\title{
Placental Malaria Associated With Low Birth Weight among HIV-Infected Pregnant Women on Cotrimoxazole Prophylaxis in Jos, Nigeria
}

\author{
Murna Ahmed Ali ${ }^{1 *}$, Joseph Aje Anejo-Okopi ${ }^{1,2}$, Stephen Oguche ${ }^{2,3}$, Jonah Musa ${ }^{2,4}$, Ocheme Julius Okojokwu1, \\ Patricia Manko Lar ${ }^{1}$, Suleiman Mohammed Maryam5, Ediga Bede Agbo ${ }^{5}$ \\ 1Department of Microbiology, Faculty of Natural Sciences, University of Jos, Jos, Nigeria \\ ${ }^{2}$ AIDS Prevention Initiative in Nigeria, Jos University Teaching Hospital, Jos, Nigeria \\ ${ }^{3}$ Department of Paediatrics, Faculty of Medical Sciences, University of Jos, Jos, Nigeria \\ ${ }^{4}$ Department of Obstetrics and Gynaecology, Faculty of Medical Sciences, University of Jos, Jos, Nigeria \\ ${ }^{5}$ Department of Biological Sciences, Abubakar Tafawa Balewa University, Bauchi Bauchi, Nigeria
}

\section{*Corresponding Author \\ Murna Ahmed Ali}

\section{Article History}

Received: 31.08.2019

Accepted: 13.09 .2019

Published: 15.10 .2019

\begin{abstract}
The effectiveness of prophylaxis with cotrimoxazole against placental and infants' malaria parasitaemia, anaemia and birth weight was assessed among HIV-infected pregnant women in Jos. Thick and thin blood smears of placental and cord blood were examined for malaria parasitaemia while other birth outcomes were taken at delivery. Out of the 135 HIV-infected pregnant women exposed to cotrimoxazole (CTX) Group A and 100 to sulfadoxine-pyrimethamine (SP) Group B for prophylaxis, 102(75.6\%) and 93(93.0\%) in groups A and B respectively were followed-up to delivery. Three (2.94\%) infants in group A and $1(0.98 \%)$ in group B were preterm deliveries/stillbirths. No maternal death was recorded throughout the study. Placental malaria was $5.9 \%$ among group $A$ and $7.5 \%$ among group B. No malaria parasites were detected in the cord blood of group A infants while $3.2 \%$ of group B infants were infected with the parasites. Mean placental malaria parasitaemia was low $(<2000 a s p / \mu l)$ throughout the study, with 1266.6 and 0 asp/ $\mu \mathrm{l}$ in the placenta and cord blood respectively of Group A and 1157.14 and 933.3 asp/ $\mu$ respectively, in group B. Severe infants' anaemia $(\mathrm{PCV}<25)$, was higher among study population who took SP for prophylaxis compared to those who took CTX $8(8.60 \%)$ and $6(5.90 \%)$ respectively. The means of all other birth outcomes were within normal ranges except for the placental and cord blood PCVs which were slightly lower than the normal ranges (36-47\% and 42-60\% respectively). Low birth weight (LBW) was observed among infants in SP group (B) than CTX group (A). Placental malaria was significantly associated with cord blood malaria, infant birth weight, and cord blood PCV. Prophylaxis with CTX effectively reduced placental and infants' malaria parasitaemia compared to the standard SP. Continuous monitoring of haemoglobin status of HIV-infected pregnant women with low CD4+ count should be strengthened for promptly treatment of anaemic patients.
\end{abstract}

Keywords: Placental, Malaria, cotrimoxazole, HIV-positive, pregnant women, new-born child.

\section{INTRODUCTION}

The importance of placental malaria as a complication of malaria in pregnancy has long been recognised, particularly in areas of stable malaria transmission. Plasmodium-infected erythrocytes accumulate in the intervillous space in the placenta, causing histologic changes including leukocyte-induced damage to the trophoblastic basement membrane of the placenta [1]. This often results in placental insufficiency and poor nutrition for the foetus, leading to Low Birth Weight, an important risk factor for infant mortality [2].

The World Health Organization recommended Cotrimoxazole prophylactic treatment (CPT) for prevention of opportunistic infections in adults, children and HIV-exposed infants while they remain at risk of HIV acquisition through breastfeeding [3]. The benefits of CPT have been well established in adults and HIV-infected children but limited information exists among HIV-infected pregnant women and HIV-exposed, uninfected infants, including whether CPT offers protection against malaria. Despite its prophylactic action against malaria and usefulness in promoting healthier pregnancies [4-6], the use of Sulfadoxine Pyrimethamine

Copyright @ 2019: This is an open-access article distributed under the terms of the Creative Commons Attribution license which permits unrestricted use, distribution, and reproduction in any medium for non commercial use (NonCommercial, or CC-BY-NC) provided the original author and source are credited. 
(SP) among HIV-infected pregnant women along with Cotrimoxazole (CTX) prophylaxis has been discouraged by the WHO due to increased side-effects thought to be associated with the concurrent use of the two. The WHO had instead, recommended the use of Cotrimoxazole alone for the dual purposes of prophylaxis against both malaria and opportunistic bacteria in HIV-infected persons with low CD4+ count [7]. Despite the increase in PMTCT programs available to pregnant women characterized by low uptake by HIV infected women, there is increase in spontaneous abortion, still birth, infant mortality, intrauterine growth retardation, low birth weight and preterm delivery due to comorbidities; however prophylactic treatment for pregnant mothers has great benefits to the child health. Without access to treatment, approximately one third of infants will die before one year of age, and half will die by their second birthday. Perinatally infected children are at particular risk of death between two and six months of age. Pregnancy associated malaria (PAM) causes adverse pregnancy and birth outcomes owing to Plasmodium falciparum accumulation in the placenta. However there is paucity of data on the impact of the drug on Placental malaria parasitaemia in HIV-infected pregnant women from the sub-Saharan region known to be the worst hit by malaria. There are few available cross-sectional studies from other sub-Saharan countries, but we are not yet aware of any longitudinal study that has reported this in Nigeria. The study aimed to find out the impact of prophylaxis with cotrimoxazole vs SP on the prevalence and intensity of placental malaria, anaemia and low birth weight in HIV-infected pregnant women in Jos, North-central Nigeria.

\section{Materials ANd Methods}

The study population consisted of 235 HIV-infected pregnant women visiting antenatal care units of Bingham University Teaching Hospital (BHUTH), Faith Alive Foundation Hospital (FAFH) and Plateau State Specialist Hospital (PSSH) all in Jos, Plateau state. Women in their second trimesters (from week 13) who fulfilled the inclusion criteria and consented for the study were enrolled. These were divided into two groups (A and B) depending on their CD4+ count. Group A comprises those with $C D 4+$ count of $\leq 350$ cells $/ \mathrm{cm}^{3}$ while Group B comprises those with CD4+ count of $>350 \mathrm{cells} / \mathrm{cm}^{3}$. Group A population were given daily Cotrimoxazole $(960$ $\mathrm{mg}$ ) for prophylaxis, while Group B (control group) had 3 doses of $525 \mathrm{mg} \mathrm{SP}$, since this is generally being used as the standard care against malaria in pregnancy $[8,5]$. Placental and cord blood were collected immediately after delivery for the determination of malaria parasitaemia and PCV. Placental and infants' weights were determined at birth using an electronic weighing balance, while placental and foetal lengths, head and mid-arm circumference were determined using a measuring tape. Thin and thick blood smears were prepared and viewed under the microscope for malaria parasitaemia according to the method described [3]. At least 200 WBCs in several microscopic fields were usually counted with the associated parasites, after which the parasite density was estimated using already described formula $[9,3,4]$.

\section{Determination of Packed Cell Volume (PCV)}

About three- quarters of a plain capillary tube was filled with fresh well mixed whole blood from an anticoagulated EDTA bottle. The capillary tube was then sealed at the unfilled end using a sealant. The filled capillary tube was then slotted in one of the numbered slots of the Microhaematocrit rotor, with the sealed end against the rim gasket (to prevent breakage). The number of the slot was usually written on the patient's form. It was then centrifuged for $5 \mathrm{~min}$ at 12,000rpm. The PCV was determined according to the method described by Bull et al. [10].

\section{Data Analysis}

Data generated were analysed using SPSS statistical software for Windows 11.0 (SPSS Inc. USA) and results presented on tables and charts. The $5 \%$ level of probability $(P<0.05)$ was considered significant with a confidence interval of $95 \%$.

\section{RESULT}

\section{Placental malaria and birth outcomes of HIV-infected pregnant women in relation to Prophylactic Treatment}

The means of most birth outcomes were within normal ranges except for Placental and cord blood PCV as shown below (Figure 2). Prevalence of placental malaria was lower among the women that was treated with CTX (5.9\%) than the SP $(7.5 \%)$, with the highest being $1600 \mathrm{asp} / \mathrm{ml}$ with mean value of 1266.67 (CTX) and $1157.17(\mathrm{SP})$. No malaria parasites were detected in the cord blood of infants from the CTX group, whereas 3/100 (3.0\%) infants of SP group harboured the parasites in their cord blood. All of the 3 cases with malaria positive cord blood had the parasites in their placentas. We observed the lowest placental malaria parasitaemia (500asp/ml) among the SP group while the highest (1500-2000 asp/ml) was observed in both groups (Figure 1). 
Table-2: Infants' anthropometry of study population in relation to prophylactic drugs used

\begin{tabular}{|c|c|c|c|c|c|c|}
\hline \multirow{3}{*}{ Parameter } & \multicolumn{5}{|c|}{ Study Group } & \multirow{3}{*}{ Normal Value } \\
\hline & \multicolumn{2}{|c|}{$A \quad(n=135, N L F=33)$} & \multicolumn{3}{|c|}{$(n=100, N L F=7)$} & \\
\hline & $F$ & $\%$ & $F$ & & $\%$ & \\
\hline \multicolumn{7}{|l|}{ Infant length $(\mathrm{cm})$} \\
\hline$<45$ & & 11 & 8.1 & 7 & 7 & $\geq 45 \mathrm{~cm}$ \\
\hline$\geq 45$ & & 91 & 67.4 & 87 & 87 & \\
\hline Valid no. & & 102 & 75.6 & 93 & 93 & \\
\hline \multicolumn{7}{|l|}{ Infant HC (cm) } \\
\hline$<33$ & & 11 & 8.1 & 9 & 9 & $\geq 33 \mathrm{~cm}$ \\
\hline$\geq 33$ & & 91 & 67.4 & 85 & 85 & \\
\hline Valid no. & & 102 & 75.6 & 93 & 93 & \\
\hline \multicolumn{7}{|l|}{ Infant MUAC(cm) } \\
\hline$<9.5$ & & 3 & 2.2 & 1 & 1 & $\geq 9.5 \mathrm{~cm}$ \\
\hline$\geq 9.5$ & & 99 & 73.3 & 92 & 92 & \\
\hline Valid no. & & 102 & 75.6 & 93 & 93 & \\
\hline \multicolumn{7}{|c|}{ Placental weight (kg) } \\
\hline$<0.4$ & & 8 & 5.9 & 1 & 1 & $\geq 0.4 \mathrm{~kg}$ \\
\hline$\geq 0.4$ & & 94 & 69.6 & 92 & 92 & \\
\hline Valid no. & & 102 & 75.6 & 93 & 93 & \\
\hline \multicolumn{7}{|c|}{ Placental length(cm) } \\
\hline$<15$ & & 0 & 0 & 0 & 0 & $\geq 15 \mathrm{~cm}$ \\
\hline$\geq 15$ & & 102 & 75.6 & 93 & 93 & \\
\hline Valid no. & & 102 & 75.6 & 93 & 93 & \\
\hline
\end{tabular}

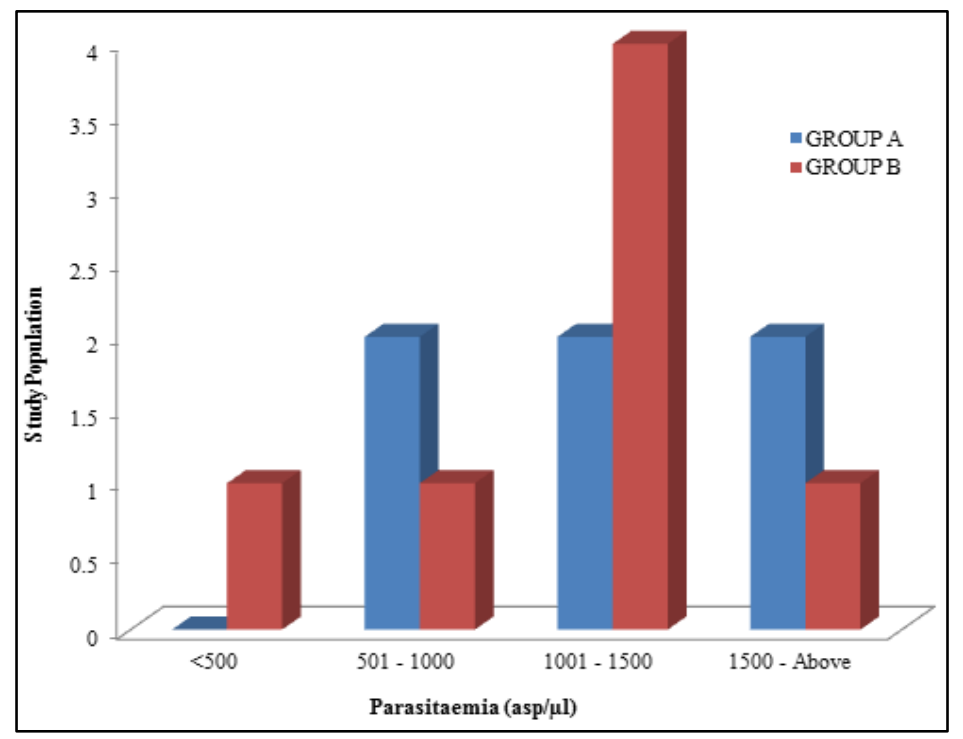

Fig-1: Placental malaria parasitaemia among HIV-infected pregnant women in relation to Prophylactic Treatment

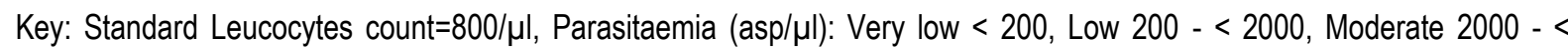
20,000 , High parasitaemia $>20,000$

\section{Placental and infants' anaemia among HIV-infected pregnant women in relation to antimalarial Drugs used for prophylaxis}

Severe infants'anaemia (PCV<25), was higher among study population who took SP for prophylaxis compared to those who took CTX [8(8.60\% and 6(5.90\% respectively). Most other study participants had mild-moderate anaemia (PCV=26-41) as shown on the chart. Severe infant's anaemia was found in $12.5 \%$ of women with placental malaria among both groups after prophylaxis (Figure 2). The placental blood PCV of study population. There were more women with severe placental anaemia (PCV $<25 \%)$ among the SP compared to the CTX group (12.0\% and $9.6 \%$ respectively). The rest had mild-moderate placental anaemia, while $37(36.27 \%)$ in the CTX group and $29(31.18 \%)$ in the SP group had normal placental PCVs (Figure 3). Infant's PCV (\%), was significantly $(P<0.05)$ associated with placental PCV, infant and placental length and MUAC $(P<0.05)$ among the CTX group. 


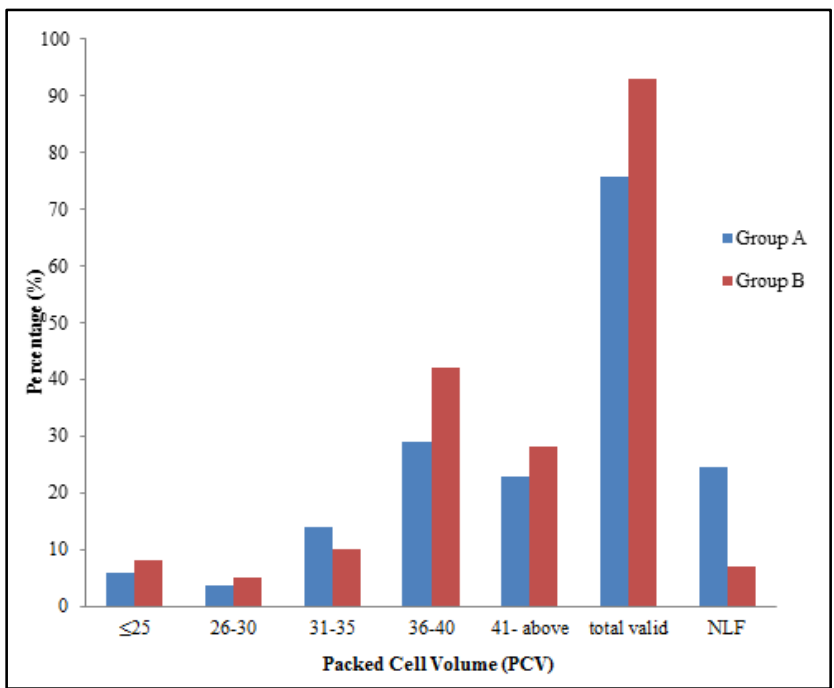

Fig-2: Packed Cell Volume (PCV) of Infants' Cord blood in relation to malaria prophylaxis taken by the mother

Where: Normal cord PCV $=42 \%$ and above, Severe anaemia $=\leq 25 \%$, Moderate anaemia $=26-35 \%$ and Mild anaemia $=36-41 \%$.

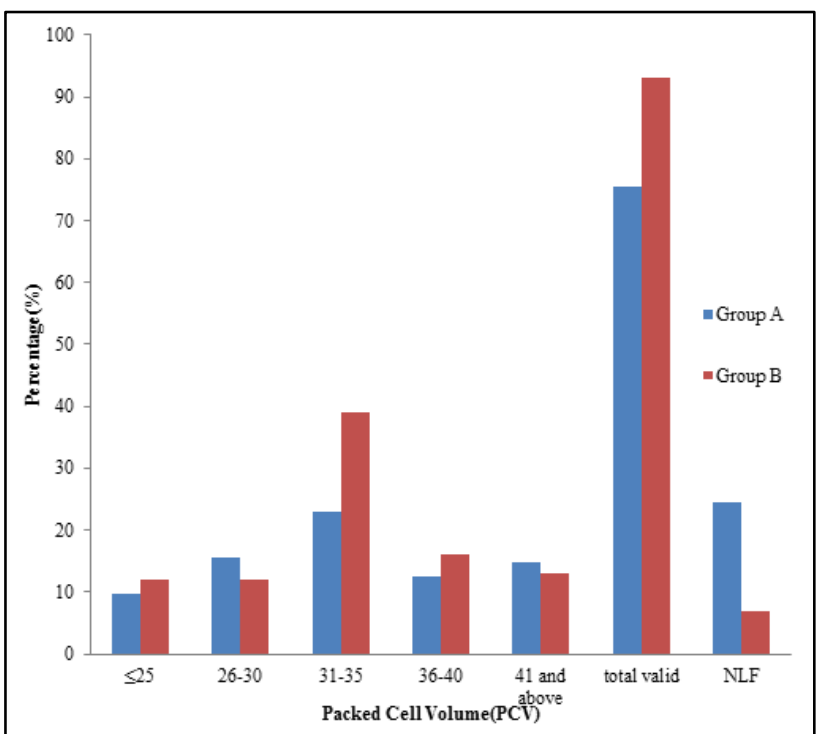

Fig-3: Packed Cell Volume (PCV) of Placental blood in relation to antimalarial prophylaxis

Where: Normal Placental PCV $=36$ and above, Severe anaemia $=\leq 25 \%$ Moderate anaemia $=26-30 \%$, Mild anaemia $=31-35 \%$

\section{Birth weights and anthropometry of infants of HIV-infected pregnant women in relation to prophylactic drugs}

Prevalence of LBW was higher among the CTX group than the SP (15.70\% and $10.8 \%$ respectively). Three $(17.6 \%)$ of the neonates with malarious placentas in group A and $6(46.2 \%)$ in group B, had LBW and Infants' birth weight (IBW) was significantly associated with placental malaria $(P<0.05)$ throughout the study (Figure 4). The anthropometric measurements of infants and their placentas are presented in Table 1. There were more infants with lower anthropometry in the CTX group than the SP group as shown on the table. All the placentas in the study were of normal lengths $(\geq 15 \mathrm{CM})$. Statistically, infant weight was significantly associated with placental lengths, infant length $\mathrm{HC}$ and MUAC $(\mathrm{P}<0.05)$. 


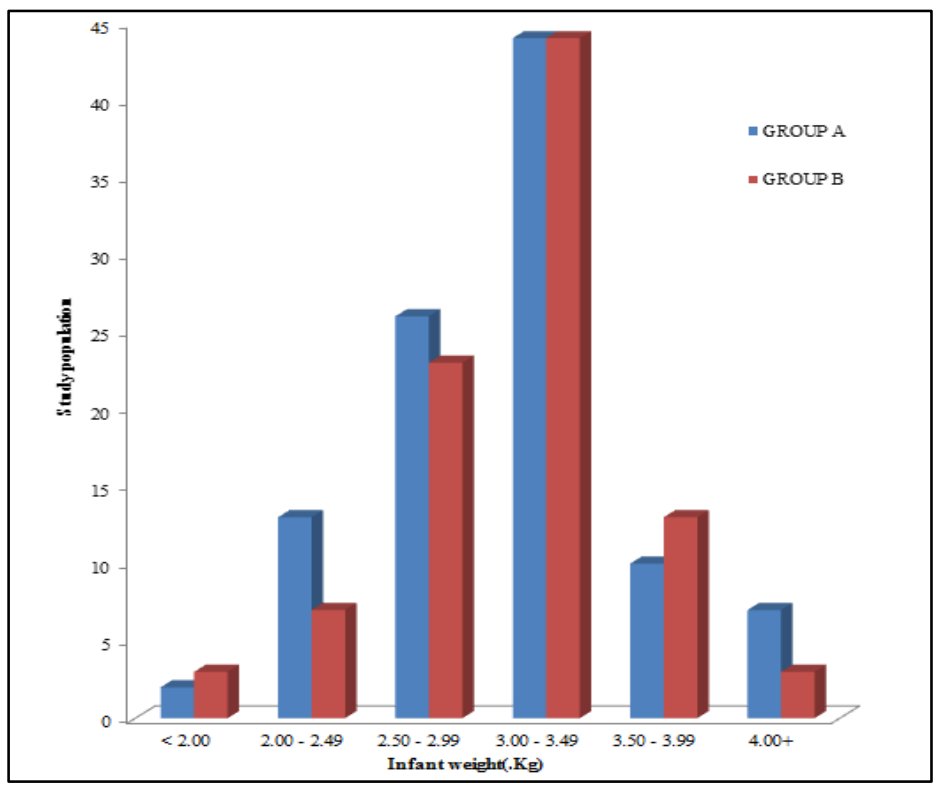

Fig-4: Infant Birth Weight in relation to antimalarial prophylaxis taken by the mother Where: Normal birth weight $=2.5-3.5 \mathrm{Kg}$, Low birth weight $=<2.5 \mathrm{Kg}$

\section{Discussion}

Malaria infection among those pregnant women with HIV increases is common, as both cause substantial morbidity and mortality due to decreased immunity after HIV infection [11]. It reported that concurrent HIV infection and malaria during pregnancy is a very serious problem for both maternal and child health $[12,13]$, and pregnant women infected with HIV are more likely to have malaria episodes [14] and the coinfection has a synergistic effect, particularly on severe anaemia, which increases maternal mortality [11].

The low prevalence of placental malaria among women with CTX prophylaxis points to the effectiveness of the drug in controlling malaria parasites even in the face of compromised immunity. It also goes further to confirm the hypothesis that suggests its usefulness for dual prophylaxis. This is even as the parasitaemia was also low and comparable to that found among those who used SP for antimalarial prophylaxis.

The $5.9 \%$ vs $7.5 \%$ prevalence of placental malaria recorded in pregnant women with CTX and SP prophylaxis respectively in this study is lower than the $20.3 \%$ vs $24.6 \%$ as reported earlier [15] from Burkina Faso. Another study reported higher percentage than $0.97 \%$ who used CTX for prophylaxis in Benin republic [16]. The higher effectiveness of CTX in reducing placental malaria compared to $\mathrm{Sp}$ in our study is consistent with their findings $[17,15,16]$. The effectiveness of CTX over SP against malaria may be related to the daily use of CTX which ensures the blood level of CTX, as against an intermittent use of SP for prophylaxis.

The lack of detectable malaria parasitaemia in the cord blood of infants whose mothers took CTX is noteworthy. This suggests the effectiveness of the drug in preventing the grievous consequences of infant malaria which is an important risk factor to infant mortality and morbidity. Previous reports had established the usefulness of prophylaxis in reducing placental malaria [18, 19], which is consistent in our findings.

The higher frequency of severe anaemia reported among the SP group compared to the CTX $(12.0 \%$ and $9.6 \%$ respectively), is contrary to the report of a similar study from Burkina Faso by Klement et al. [15], in which there were more severe anaemic cases with CTX use than SP $(10 \%$ vs $4 \%$ respectively). Both HIV and antifolate drugs like CTX and SP have been associated with anaemia in other reports $[11,17,20]$. This, in addition to the effect of malaria parasites may have given rise to the high number of severely anaemic infants recorded in this study.

The mean birth weights of both CTX and SP groups were similar in this study. These findings are consistent with what has been reported in similar studies [15]. The efficacy of the treatment regimens in preventing maternal infection, placental malaria, and low birth weight increased with gravidity. This finding confirms the high burden of malaria for women with low gravidity in areas with intense parasite transmission. The higher prevalence of LBW among the CTX compared to the SP $(15.70 \%$ and $10.8 \%$ respectively) is similar to that reported from the Burkina Faso's study (19.8\% in the CMX group and $17.0 \%$ in the IPT-SP group). Low birth weight is known to be an important risk factor for infant mortality. In this study, placental malaria significantly $(P<0.05)$ associated with IBW. Chronic infection of the placenta may lead to placental insufficiency, resulting in. Although several other factors have been associated with LBWs, destruction of the placenta by malaria parasites causes placental insufficiency resulting in poor nutrition for the infant and hence poor or retarded intrauterine growth in sub-Saharan Africa [21, 22] Furthermore, low birth weight impairment was due to high 
parasitaemia, these findings underscore the importance of implementing efficacious prevention, prompt diagnosis and highly effective anti-malarial treatment during pregnancy $[23,6]$. Several studies also showed an increased prevalence of low birth weight $(<2500 \mathrm{~g})$, preterm delivery and intrauterine growth retardation in coinfected women [24]. Co-infection with malaria is also associated with an increased HIV load, increasing the risk for mother-to-child transmission of HIV [25]. Brentlinger et al. [26] observed that concurrent management of malaria and HIV infection in pregnancy in sub-Saharan Africa is still very challenging, and suggested that medications against the two infections must be administered with precaution due interactions and overlapping toxicity. HIV-positive women who are not taking co-trimoxazole prophylaxis for opportunistic infections can take a monthly dose of SP, Co-trimoxazole has been shown to decrease malaria morbidity in children and HIV-infected adults [27-29]. Denoeud-Ndam et al. [16] reported that co-trimoxazole alone provided adequate protection against malaria in HIV infected pregnant women.

\section{CONCLUSION}

Malaria is the commonest tropical parasite-transmitted disease during pregnancy, and, it requires rapid diagnosis and appropriate treatment to avoid complications for the woman and her foetus. There is the need for safe, new, effective antimalarial agents, due the growing resistance of $\mathrm{P}$. falciparum to intermittent preventive treatment in pregnancy (IPTp), as prophylactic chemotherapy is the main tool for controlling malaria during pregnancy. Special attention should be paid to diagnosis and the management of malaria co-infection with HIV in pregnancy, and studies of antimalarial drugs resistance and interactions between antiretroviral when co-administered are essential. Our study confirms that prophylaxis with Cotrimoxazole yields lower placental and infants' malaria prevalence and parasitaemia compared to the standard drug SP. The prevalence of severe anaemia and LBW are similar among the two groups, but more needs to be done in closely managing HIV-infected pregnant women on prophylaxis in order to curb the harmful consequences of anaemia in that population.

\section{REFERENCES}

1. Ouédraogo, A., Tiono, A. B., Diarra, A., Bougouma, E. C. C., Nébié, I., Konaté, A. T., \& Sirima, S. B. (2012). Transplacental transmission of Plasmodium falciparum in a highly malaria endemic area of Burkina Faso. Journal of tropical medicine, 2012.

2. Georgian, I., Matthew, O., \& Nte, N. I. (2017). Prevalence and effect of malaria in pregnancy among antenatal women in Ebonyi State, Nigeria. International Research Journal of Public and Environmental Health, 4(8), 177-183.

3. World Health Organization-WHO. (1999). Bench Aids for the Diagnosis of Malaria infections. PP.24.

4. Garner, P., \& Gülmezoglu, A. M. (2006). Drugs for preventing malaria in pregnant women. Cochrane database of systematic reviews, (4).

5. World Health Organization. (2007). Malaria in pregnancy: guidelines for measuring key monitoring and evaluation indicators.

6. World Health Organisation-WHO. (2012). World malaria report 2012. ww.who.int/malaria/publications/world_malaria_report_2012/wmr2012_no_profiles.pdf PP35-45.

7. World Health Organization-WHO. (2006). Guidelines on co-trimoxazole prophylaxis for HIV infections among children, adolescents and adults in resource-limited settings: recommendations for a public health approach. WHO Geneva: 49pp.

8. World Health Organization-WHO/UNICEF. (2003). The Africa Malaria Report - 2003

9. Trape, J.F. (1995). Rapid evaluation of malaria parasite density and standardization of thick smear examination for epidemiological investigations. Trans. R. Soc. Trop. Med. Hyg, 79(2):181-184.

10. Bull, B.S., Koepke, J.A., Simson, E., van Assendelft, O.W. (2000). Procedure for Determining Packed Cell Volume by the Microhaematocrit method.Approved Standard 3rd edition. Clinical and Laboratory Standard Institute (CLSI), 20(19): 25.

11. Ayisi, J. G., Van Eijk, A. M., Ter Kuile, F. O., Kolczak, M. S., Otieno, J. A., Misore, A. O., ... \& Nahlen, B. L. (2003). The effect of dual infection with HIV and malaria on pregnancy outcome in western Kenya. Aids, 17(4), 585-594.

12. Meshnick, S. R., Mwapasa, V., \& Rogerson, S. J. (2006). Protecting pregnant women from malaria in areas of high HIV infection prevalence. The Journal of infectious diseases, 194(3), 273-275.

13. Takem, E. N., \& D'Alessandro, U. (2013). Malaria in pregnancy. Mediterranean journal of hematology and infectious diseases, 5(1).

14. Van Eijk, A. M., Ayisi, J. G., Ter Kuile, F. O., Otieno, J. A., Misore, A. O., Odondi, J. O., .. \& Nahlen, B. L. (2004). Effectiveness of intermittent preventive treatment with sulphadoxine-pyrimethamine for control of malaria in pregnancy in western Kenya: a hospital-based study. Tropical medicine \& international health, 9(3), 351-360.

15. Klement, E., Pitché, P., Kendjo, E., Singo, A., D'Almeida, S., Akouete, F., ... \& Caumes, E. (2013). Effectiveness of cotrimoxazole to prevent Plasmodium falciparum malaria in HIV-positive pregnant women in sub-Saharan Africa: an open-label, randomized controlled trial. Clinical infectious diseases, 58(5), 651-659.

16. Denoeud-Ndam, L., Zannou, D. M., Fourcade, C., Taron-Brocard, C., Porcher, R., Atadokpede, F., ... \& Girard, P. M. (2014). Cotrimoxazole prophylaxis versus mefloquine intermittent preventive treatment to prevent malaria in HIV-infected pregnant women: two randomized controlled trials. JAIDS Journal of Acquired Immune Deficiency Syndromes, 65(2), 198-206.

17. Tiono, A. B., Ouedraogo, A., Bougouma, E. C., Diarra, A., Konaté, A. T., Nébié, I., \& Sirima, S. B. (2009). Placental malaria and low birth weight in pregnant women living in a rural area of Burkina Faso following the use of three preventive treatment regimens. Malaria journal, 8(1), 224.

18. Uneke, C. J. (2008). Impact of placental Plasmodium falciparum malaria on pregnancy and perinatal outcome in sub-Saharan Africa: part III: placental malaria, maternal health, and public health. The Yale journal of biology and medicine, 81(1), 1. 
19. Izuka, E. O., Ugwu, E. O., Obi, S. N., Ozumba, B. C., Nwagha, T. U., \& Obiora-Izuka, C. E. (2017). Prevalence and predictors of placental malaria in human immunodeficiency virus-positive women in Nigeria. Nigerian journal of clinical practice, 20(1), 31-36.

20. Lesi, F. E. A., Mukhtar, M. Y., Iroha, E. U., \& Egri-Okwaji, M. T. C. (2010). Clinical presentation of congenital malaria at the Lagos University Teaching Hospital. Nigerian journal of clinical practice, 13(2).

21. Malhotra, I., Mungai, P., Muchiri, E., Kwiek, J. J., Meshnick, S. R., \& King, C. L. (2006). Umbilical cord-blood infections with Plasmodium falciparum malaria are acquired antenatally in Kenya. The Journal of infectious diseases, 194(2), 176-183.

22. Obiajunwa, P. O., Owa, J. A., \& Adeodu, O. O. (2005). Prevalence of congenital malaria in Ile-Ife, Nigeria. Journal of Tropical Pediatrics, 51(4), 219-222.

23. McGready, R., White, N. J., \& Nosten, F. (2011). Parasitological efficacy of antimalarials in the treatment and prevention of falciparum malaria in pregnancy 1998 to 2009: a systematic review. BJOG: An International Journal of Obstetrics \& Gynaecology, 118(2), 123-135.

24. Ter Kuile, F. O., Parise, M. E., Verhoeff, F. H., Udhayakumar, V., Newman, R. D., Van Eijk, A. M., ... \& Steketee, R. W. (2004). The burden of co-infection with human immunodeficiency virus type 1 and malaria in pregnant women in sub-saharan Africa. The American journal of tropical medicine and hygiene, 71(2_suppl), 41-54.

25. Brahmbhatt, H., Sullivan, D., Kigozi, G., Askin, F., Wabwire-Mangenm, F., Serwadda, D., ... \& Gray, R. (2008). Association of HIV and malaria with mother-to-child transmission, birth outcomes, and child mortality. JAIDS Journal of Acquired Immune Deficiency Syndromes, 47(4), 472-476.

26. Brentlinger, P. E., Behrens, C. B., \& Micek, M. A. (2006). Challenges in the concurrent management of malaria and HIV in pregnancy in sub-Saharan Africa. The Lancet infectious diseases, 6(2), 100-111.

27. Hamel, M. J., Greene, C., Chiller, T., Ouma, P., Polyak, C., Otieno, K., ... \& Brooks, J. T. (2008). Does cotrimoxazole prophylaxis for the prevention of HIV-associated opportunistic infections select for resistant pathogens in Kenyan adults?. The American journal of tropical medicine and hygiene, 79(3), 320-330.

28. Dibua, U. M., Badger-Emeka, L., \& Ugonabo, J. A. (2013). HIV and malaria co-infection: Their combined effects on pregnancy outcomes in Anambra State, Southeast Nigeria. International Journal of Medicine and Medical Sciences, 5(10), 438-449.

29. González, R., Rupérez, M., Sevene, E., Vala, A., Maculuve, S., Bulo, H., \& Menendez, C. (2017). Effects of HIV infection on maternal and neonatal health in southern Mozambique: A prospective cohort study after a decade of antiretroviral drugs roll out. PloS one, 12(6), e0178134. 